\title{
Kompetenzanforderungen an Führungskräfte von agilen Softwareentwicklungsteams
}

\author{
Sarah Anke ${ }^{1}$ Tobias Ringeisen ${ }^{2}$ \\ Angenommen: 2. Januar 2021 / Online publiziert: 22. Januar 2021 \\ (c) Der/die Autor(en) 2021
}

\section{Zusammenfassung}

Auf Basis des Great Eight-Kompetenzrahmens stellt dieser Beitrag der Zeitschrift Gruppe. Interaktion. Organisation. (GIO) eine Studie zu den Kompetenzanforderungen an Projektleiter*innen in hochdigitalisierten Arbeitsumgebungen der agilen Softwareentwicklung vor und definiert den zugehörigen Führungserfolg. Es wurden elf Einzelinterviews mit Expert*innen eines internationalen IT-Dienstleisters durchgeführt, die verschiedene Rollen im Kontext der Softwareentwicklung ausfüllten. Die Interviews wurden mittels qualitativer Inhaltsanalyse anhand eines Kategoriensystems computergestützt ausgewertet. Es konnte ein Anforderungsprofil identifiziert werden, das 20 Kompetenzfacetten im Sinne leistungsrelevanter Verhaltensweisen umfasst. Eine bis drei Kompetenzfacetten ließen sich jeweils einer von 12 übergeordneten Kompetenzdimensionen zuordnen, wobei jede Domäne der Great Eight durch mindestens eine Kompetenzdimension repräsentiert war. Die Domänen Leading and Deciding (Motivationsfähigkeit; Mitarbeiterförderung), Interacting and Presenting (Kommunikationsfähigkeit; Konfliktfähigkeit) sowie Analyzing and Interpreting (Kenntnisse IT-Branche; Überblickswissen Softwareentwicklung; agile Arbeitsmethoden) umfassten jeweils zwei bzw. drei Kompetenzdimensionen. Die restlichen fünf Domänen wurden jeweils durch eine Kompetenzdimension abgebildet (Supporting und Cooperating: Teamfähigkeit; Creating and Conceptualizing: Offenheit; Organizing and Executing: Zeit- und Ressourcenmanagement; Adapting and Coping: Proaktiver Umgang mit Fehlern; Enterprising and Performing: Begeisterungsfähigkeit für Softwareentwicklung). Die Domänen Analyzing and Interpreting sowie Enterprising and Performing bilden Kompetenzanforderungen ab, die spezifisch für den Arbeitskontext der agilen Softwareentwicklung sind. Führungserfolg liegt vor, wenn lauffähige Software ressourceneffizient nach Kundenwünschen entwickelt wird und die Teammitglieder gleichzeitig eine hohe Arbeitszufriedenheit berichten. Zusammenfassend lassen sich Kompetenzanforderungen an Projektleiter*innen in digitalisiert und agil agierenden Softwareentwicklerteams anhand der Great Eight konzeptualisieren, wobei das Anforderungsportfolio vier tätigkeitsfeldspezifische und acht kontextunabhängige Kompetenzdimensionen vereint, die sich jeweils anhand relevanter Verhaltensweisen für den Führungsalltag definieren lassen.

Schlüsselwörter Kompetenzanforderungen $\cdot$ Führungskräfte $\cdot$ Agile Arbeitsmethode $\cdot$ Softwareentwicklung . Personalauswahl

Sarah Anke, B.A.

sarah.anke@gmx.de

Prof. Dr. Tobias Ringeisen

tobias.ringeisen@hwr-berlin.de
04275 Leipzig, Deutschland

2 Berlin School of Economics and Law, Alt-Friedrichsfelde 60, 10315 Berlin, Deutschland 


\title{
Competence requirements for project managers in agile software development
}

\begin{abstract}
Based on the Great Eight competence framework this article of Gruppe. Interaktion. Organisation. (GIO) analyzed competency requirements and leadership success criteria of project managers, who supervise agile software development teams. Eleven experts from an international IT company, who fulfilled various roles in agile software development, were interviewed individually. The interviews were analyzed by means of qualitative content analysis using a computer-aided category system. In terms of performance-relevant behaviors, 20 competence facets with 12 superordinate competence dimensions could be identified. One to three dimensions represented each domain of the Great Eight. The domains Leading and Deciding (motivating others; promoting others), Interacting and Presenting (target-appropriate communication; conflict management) as well as Analyzing and Interpreting (IT knowledge; software development expertise; agile working methods) comprised two or three dimensions, respectively. The remaining five domains included one dimension each (Supporting und Cooperating: team work skills; Creating and Conceptualizing: openness; Organizing and Executing: resource management; Adapting and Coping: dealing with mistakes; Enterprising and Performing: enthusiasm for software development). Analyzing and Interpreting as well as Enterprising and Performing contained competency requirements, which are specific for agile software development. Leadership success is achieved when executable software is developed in a resource-efficient manner according to customer requirements, while the development team reports high job satisfaction. In summary, the competence requirements of project managers in agile development teams may be conceptualized by means of the Great Eight framework. The portfolio consists of four context-specific and eight cross-contextual competence dimensions, each of which may be operationalized by means of specific behaviors.
\end{abstract}

Keywords Competence requirements $\cdot$ Leaders $\cdot$ Agile working methods $\cdot$ Software development $\cdot$ Personnel selection

\section{Einleitung}

Es stehen verschiedene Rahmenmodelle zur Verfügung, um Kompetenzanforderungen an Führungskräfte in informationstechnologisch geprägten Arbeitsumgebungen zu konzeptualisieren (z. B. European Committee for Standardization (CEN) 2016; Deutsche Gesellschaft für Personalführung 2016; Kluzer und Pujol Priego 2018). Diese Kompetenzrahmen ergeben sich aus den Geschäftsprozessen der entsprechenden Arbeitsfelder und repräsentieren die Sicht der betrieblichen Praxis. Die Auflösung und Spezifikation der abgebildeten Kompetenzdomänen sowie der darunter gebündelten Kompetenzdimensionen variiert je nach Rahmenkonzept, was eine theoretische Verortung, empirische Überprüfung und Vergleichbarkeit der Modelle erschwert.

Finden stattdessen branchenübergreifende und kriterienbasiert validierte Kompetenzrahmen Anwendung, um Anforderungsprofile für Führungskräfte zu entwickeln, so ergeben sich eine Reihe von Vorteilen (Blickle 2019; Schmidt-Rathjens 2007; Sonntag und Schmidt-Rathjens 2005). Erstens weisen übergeordnete Kompetenzrahmen wie die Great Eight (z.B. Bartram 2005) mit acht Domänen und mehr als 100 Kompetenzfacetten eine hohe Auflösung auf, um die gesamte Bandbreite potenzieller Determinanten der Führungsleistung differenziert und valide abzubilden. Zweitens lässt sich leicht prüfen, ob alle theoretisch zu erwartenden Kompetenzdomänen vertreten sind, was betriebsintern die Vergleichbarkeit der Anforderungsprofile bei Stellenbewertungen und die Konzeption bereichsübergreifender Auswahlverfahren erleichtert. Dies gilt beispielsweise, wenn Führungskräfte Projektteams leiten, von denen einige virtuell, andere hingegen vor Ort zusammenarbeiten (Krumm et al. 2016). Drittens lässt sich die je nach Arbeitsumgebung die variierende Bedeutung von universellen Kompetenzdimensionen als auch das Auftreten kontextspezifischer Anforderungen bestimmen.

Trotz vielfältiger Handlungsempfehlungen für die Praxis gibt es bisher wenig Forschung zu den Kompetenzanforderungen, mit denen Projektleiter*innen in hochdigitalisierten Arbeitsumgebungen wie der Softwareentwicklung konfrontiert sind (Deutsche Gesellschaft für Projektmanagement und Gessler 2016; Geipel 2003). Um diese Forschungslücke zu schließen, untersucht die vorliegende Studie auf Basis des Great Eight-Kompetenzrahmens, über welche Kompetenzen erfolgreiche Führungskräfte im Bereich der Softwareentwicklung verfügen (sollten). Ergänzend wird analysiert, anhand welcher Kriterien sich ihr Führungserfolg definieren lässt. Die Ergebnisse dieser Anforderungsanalyse können zur Personalauswahl und späteren Personalentwicklung von Projektleiter*innen im Bereich der Softwareentwicklung sowie bei der Umstellung auf agile Arbeitsweisen in Softwareentwicklungsteams herangezogen werden (vgl. Blickle 2019; Schmidt-Rathjens 2007).

\subsection{Agile Softwareentwicklung}

Um Software zu entwickeln und implementieren, arbeiten die Beschäftigten in der IT-Branche zumeist in agilen Pro- 
jektteams zusammen (Tiemeyer 2010). Unter Agilität versteht man die Fähigkeit eines Unternehmens oder einer Arbeitsgruppe, flexibel und anpassungsfähig auf Veränderungen zu reagieren (Onpulson 2018). Die agile Arbeitsmethode entstand ab Mitte der 1990er-Jahre als Reaktion auf langsame, bürokratische Prozesse, die es den Entwicklerteams nicht erlaubten, angemessen auf die steigende Geschwindigkeit zu reagieren, die das Projektumfeld und die Produktanforderungen zunehmend charakterisierte (Preußig 2015).

Festgeschrieben wurden die folgenden 12 Prinzipien der agilen Arbeitsmethode 2001 im Manifesto for Agile Software Development, welches von einer Gruppe von 17 Softwareentwicklern und IT-Projektmanagern verfasst worden ist (Beck et al. 2001). Um (1) die Kundenzufriedenheit als oberste Priorität zu erhalten, (2) arbeiten Vertrieb und Softwareentwicklung täglich zusammen, wobei (3) dem Kunden während des gesamten Projektverlaufs in kurzen Zeitabständen lauffähige Software geliefert wird, was diesen befähigt, (4) jederzeit willkommenen Anpassungsbedarf zu melden. Es wird (5) auf unnötige Komplexität verzichtet, um die Fehleranfälligkeit und den Arbeitsaufwand zu reduzieren, während (6) der Fokus auf technischer Exzellenz und unterstützendem Design liegt. Langfristig ist agiles Arbeiten (7) nachhaltig, da Fehler kontinuierlich beseitigt und Folgeprobleme reduziert werden, was dem Kunden (8) Wettbewerbsvorteile bringt. (9) Lauffähige Software nach Kundenwünschen bildet somit das oberste Ziel und gleichzeitig den Indikator für den Projektfortschritt. Das Projektteam sollte (9) aus motivierten Personen zusammengestellt werden, die mit den benötigten Ressourcen versorgt sind und eigenverantwortlich arbeiten, was (10) einen kontinuierlichen Face-to-Face-Austausch und (11) eine regelmäßige Reflexion und Anpassung des agilen Arbeitens im Team voraussetzt. In dieser Form (12) selbstorganisiert arbeitende Teams erbringen die besten Ergebnisse.

Um die vorgestellten Prinzipien in die Praxis umzusetzen, wurden agile Vorgehensmodelle entwickelt, die sich durch eine hohe Flexibilität während der gesamten Projektlaufzeit auszeichnen (Wieczorrek und Mertens 2011). Jeder Kundenauftrag bildet dabei ein eigenes, agil gesteuertes Projekt. Die Dokumentation der Prozesse beschränkt sich auf das Nötigste. Zu Beginn wird eine Grobplanung erstellt, die auf Kundenbedürfnissen basiert, ohne die Anforderungen im Detail zu spezifizieren (Preußig 2015). Dementsprechend werden die Ziele der Umsetzung definiert, während der Weg zu deren Erreichung offenbleibt. Eine detaillierte Planung und Spezifikation erfolgt erst in demjenigen $\mathrm{Ab}$ schnitt, in dem die jeweilige Anforderung umgesetzt wird (Eckkrammer et al. 2010). Nachdem eine Iteration abgeschlossen ist, überprüft das Entwicklerteam das Ergebnis retrospektiv (Wieczorrek und Mertens 2011). So kann dem Kunden bereits zum Ende eines Zykluses funktionstüchtige Software präsentiert werden. Änderungswünsche werden im nächsten Zyklus eingearbeitet und umgesetzt. Die einzelnen Funktionalitäten wachsen solange, bis das gesamte System vollständig funktioniert (Wieczorrek und Mertens 2011). Agile Vorgehensmodelle helfen, schnell auf fortlaufend auftretende Änderungen und Anpassungsbedürfnisse im Projektverlauf zu reagieren (Eckkrammer et al. 2010). Die dafür relevanten Entscheidungen trifft vorrangig der Kunde, der durch eine kontinuierliche Beratung von den Softwareentwickler*innen unterstützt wird (Eckkrammer et al. 2010).

\subsection{Die Arbeitsweise agiler Teams}

Zur Realisierung der o.g. teambezogenen Prinzipen weisen agil arbeitende Projektteams häufig die folgenden Merkmale auf. Um die selbstorganisierte Zusammenarbeit zu begünstigen, umfassen agile Teams durchschnittlich zehn Mitarbeiter*innen, die im Allgemeinen hochqualifiziert und oftmals kulturell divers sind (Eckkrammer et al. 2010). Die Teammitglieder müssen mehr Verantwortung übernehmen als herkömmliche Arbeitsgruppen und haben eine größere Entscheidungsfreiheit, was eine höhere Identifikation mit den Aufgaben begünstigt (Gloger und Häusling 2011). Eine enge und stetige Zusammenarbeit innerhalb des Entwicklerteams steht im Zentrum der agilen Arbeitsweise (Preußig 2015). Tägliche Daily-Standup-Meetings bieten einen festen Rahmen für diesen Austausch. Sitzen die Teammitglieder örtlich verstreut, so erfolgt die Kommunikation untereinander, mit externen Spezialisten sowie mit der Führungskraft, vorwiegend anhand digitaler Medien. Der Kommunikation zwischen den Entwickler*innen und dem Kunden kommt somit eine größere Priorität zu als der Kontinuität der zugrundliegenden Prozessketten (Tiemeyer 2010).

\subsection{Konzeptualisierung von Kompetenzanforderungen an Führungskräfte}

Obwohl die agile Arbeitsweise im Bereich der Softwareentwicklung gut dokumentiert ist, bleibt bisher weitgehend unklar, über welche Kompetenzen Führungskräfte verfügen sollten, um agile Entwicklungsteams erfolgreich zu leiten (Geipel 2003). Gut dokumentiert sind hingegen Kompetenzanforderungen an Projektleiter*innen allgemein (Bohinc 2007; Deutsche Gesellschaft für Projektmanagement und Gessler 2016). Im beruflichen Kontext lassen sich Kompetenzen als ein Bündel von Verhaltensweisen definieren, die dem Erreichen von arbeitsbezogenen Zielen und/oder dem Erbringen von Leistung dienen und sich hierarchisch in Form eines Kompetenzrahmens organisieren lassen (z.B. Bartram 2005; Tett et al. 2000). Ähnliche Verhaltensweisen bilden nach diesem Verständnis als kleinste Einheit beobachtbare Kompetenzfacetten, die sich auf dieselbe übergeordnete Kompetenzdimension 
Tab. 1 Übersicht des Great Eight-Kompetenzrahmens mit zugehörigen Kompetenzdimensionen und Persönlichkeitskorrelaten nach Bartram (2005)

\begin{tabular}{|c|c|c|}
\hline Great Eight-Domäne mit Definition $^{a}$ & Zugehörige Kompetenzdimensionen ${ }^{\mathrm{a}}$ & $\begin{array}{l}\text { Persönlichkeits- } \\
\text { korrelate }^{\mathrm{a}, \mathrm{b}}\end{array}$ \\
\hline $\begin{array}{l}\text { Leading and Deciding: Initiates and directs action, takes } \\
\text { responsibility, sets goals, works autonomously }\end{array}$ & $\begin{array}{l}\text { Deciding and Initiating Action } \\
\text { Leading and Supervising }\end{array}$ & Extraversion \\
\hline $\begin{array}{l}\text { Supporting und Cooperating: Supports others, shows respect, puts } \\
\text { people first, acts with integrity, works effectively with others }\end{array}$ & Working with People (Teamfähigkeit) & Verträglichkeit \\
\hline $\begin{array}{l}\text { Interacting and Presenting } \\
\text { Communicates and networks effectively, successfully persuades and } \\
\text { influences others, relates to others }\end{array}$ & $\begin{array}{l}\text { Relating and Networking } \\
\text { Persuading and Influencing } \\
\text { Presenting and Communicating Information }\end{array}$ & Extraversion \\
\hline $\begin{array}{l}\text { Analyzing and Interpreting } \\
\text { Clear analytical thinking, quickly takes on new technology, } \\
\text { communicates well in writing; applies expertise effectively }\end{array}$ & $\begin{array}{l}\text { Writing and Reporting } \\
\text { Applying Expertise and Technology } \\
\text { Analyzing }\end{array}$ & Offenheit für Neues \\
\hline $\begin{array}{l}\text { Creating and Conceptualizing } \\
\text { Open to new ideas and experiences, seeks learning opportunities, } \\
\text { drives change and innovation, thinks strategically }\end{array}$ & $\begin{array}{l}\text { Learning and Researching } \\
\text { Creating and Innovating } \\
\text { Formulating Strategies and Concepts }\end{array}$ & Offenheit für Neues \\
\hline $\begin{array}{l}\text { Organizing and Executing } \\
\text { Works in a systematic and organized way, plans ahead, follows } \\
\text { instructions and procedures, delivers quality }\end{array}$ & $\begin{array}{l}\text { Planning and Organizing } \\
\text { Delivering Results and Meeting Customer Ex- } \\
\text { pectations } \\
\text { Following Instructions and Procedures }\end{array}$ & Gewissenhaftigkeit \\
\hline $\begin{array}{l}\text { Adapting and Coping } \\
\text { Adapts and responds well to change, manages pressure, copes with } \\
\text { setbacks }\end{array}$ & $\begin{array}{l}\text { Adapting and Responding to Change } \\
\text { Coping with Pressure and Setbacks }\end{array}$ & $\begin{array}{l}\text { Emotionale } \\
\text { Stabilität }\end{array}$ \\
\hline $\begin{array}{l}\text { Enterprising and Performing } \\
\text { Focuses on achieving work objectives, works best when impact of } \\
\text { personal efforts is obvious, seeks career advancement }\end{array}$ & $\begin{array}{l}\text { Achieving Personal Work Goals and Objectives } \\
\text { Entrepreneurial and Commercial Thinking }\end{array}$ & $\begin{array}{l}\text { Geringe } \\
\text { Verträglichkeit }\end{array}$ \\
\hline
\end{tabular}

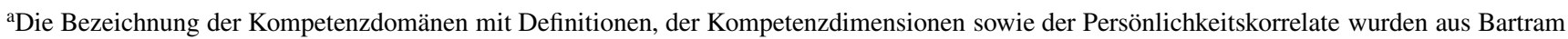
(2005, S. 1187, 1202 ff.) übernommen

${ }^{b}$ Die Persönlichkeitskorrelate entstammen dem Fünf-Faktoren-Modell der Persönlichkeit

zurückführen lassen. Die unterschiedlichen Kompetenzdimensionen clustern sich wiederum nach Ähnlichkeit in Kompetenzdomänen. Ein Satz an Kompetenzdomänen bildet auf oberster Ebene schließlich einen Kompetenzrahmen, mit dessen Hilfe sich die für ein bestimmtes Stellenprofil benötigten Kompetenzen somit bis auf die Verhaltensebene herunterbrechen lassen.

Es stehen verschiedene branchenübergreifende Rahmenmodelle zur Verfügung, um die Kompetenzanforderungen an Führungskräfte abzubilden. Die Auflösung der Kompetenzdomänen sowie der darunter gebündelten Kompetenzdimensionen variiert je nach Rahmenkonzept. Während beispielsweise im deutschsprachigen Raum viele Autor*innen Modelle mit vier Domänen (Fach-, Methoden-, Sozialund Selbstkompetenz) abgrenzen (Blickle 2019; SchmidtRathjens 2007; Sonntag und Schaper 2006; Sonntag und Schmidt-Rathjens 2005), schlagen andere eine feingliederige Struktur mit bis zu acht Domänen vor (Bartram 2005; Tett et al. 2000).

Eines der bekanntesten Rahmenmodelle zur Abbildung von Kompetenzanforderungen an Führungskräfte bilden die Great Eight (Bartram 2005; Krumm et al. 2013, 2016), welche acht Kompetenzdomänen, 20 Kompetenzdimensio- nen und 112 Kompetenzfacetten hierarchisch integrieren. Tab. 1 stellt die acht Domänen mit zugehörigen Kompetenzdimensionen und Persönlichkeitskorrelaten nach Bartram (2005) vor ${ }^{1}$. Leading and Deciding umfasst führungsbezogene Verhaltensweisen, die mit Verantwortungsübernahme, Zielsetzung und autonomer Handlungsinitiierung und -steuerung, auch bei anderen, zusammenhängen. Supporting und Cooperating bündelt Verhaltensweisen, die einen unterstützenden und bedürfnisorientierten Umgang mit anderen und eine respektvolle und effektive Zusammenarbeit ermöglichen. Interacting and Presenting charakterisiert Verhaltensweisen, die für interpersonelle Kommunikation und Networking relevant sind und beispielsweise eine aktive Beziehungsaufnahme sowie Einflussnahme auf und Überzeugung von anderen umfassen. Analyzing and Interpreting beschreibt ein schnelles analytisches Durchdringen von Problemen, was unter anderem mit dem Einbringen/ Erweitern eigener Expertise, dem Lernen neuer Technologien und geschliffener schriftlicher Kommunikation einhergeht. Creating and Conceptualizing beschreibt Verhal-

\footnotetext{
1 Auf eine Beschreibung der Kompetenzdimensionen und -facetten sowie der Persönlichkeitskorrelate wird aus Platzgründen verzichtet.
} 
tensweisen, die mit Offenheit für neue (Lern)Erfahrungen, Neugier und strategischem Denken in Verbindung stehen und das Initiieren von Veränderung und Innovation begünstigen. Organizing and Executing bündelt Verhaltensweisen, die ein planvolles, organisiertes und systematisches Herangehen an Aufgaben abbilden, das Vorgaben berücksichtigt und mit hoher Arbeitsqualität einhergeht. Adapting and Coping charakterisiert die problembezogene und emotionsbezogene Bewältigung von Stressoren und Rückschlägen. Die achte Domäne Enterprising and Performing schließlich steht für Verhaltensweisen, die dem Erreichen persönlicher und vorgegebener beruflicher Ziele dienen. Aus motivationaler Perspektive schließt diese Domäne Kompetenzfacetten wie Eigeninitiative, Ehrgeiz und Begeisterungsfähigkeit für Aufgaben und deren Zielerreichung ein.

\subsection{Kompetenzanforderungen an Führungskräfte von Projektteams}

Vergleicht man die Anforderungen an Projektleiter*innen und klassische Führungskräfte, so kommt den Domänen Leading and Deciding, Supporting and Cooperating, Interacting and Presenting sowie Analyzing and Interpreting bei den Erstgenannten eine erhöhte Bedeutung zu (Bohinc 2007; Vigenschow 2008). Einige der zugehörigen Kompetenzdimensionen und Kompetenzfacetten sind vor allem bei Projektleiter*innen im IT-Bereich relevant (Deutsche Gesellschaft für Projektmanagement und Gessler 2016; Geipel 2003).

Im Projektmanagement umfasst Leading and Deciding vor allem führungsbezogene Verhaltensweisen. Im Sinne einer Aufgabenorientierung wird von Führungskräften erwartet, projektbezogene Ziele und Visionen klar zu vermitteln, die im Projektteam anfallenden Aufgaben zu planen, deren Erledigung zu überwachen und den Mitarbeiter*innen durch Feedback regelmäßig die Qualität der Umsetzung rückzumelden. Diese Verhaltensweisen helfen Führungskräften, ihre Mitarbeiter*innen langfristig zu motivieren (Wieczorrek und Mertens 2011). Im Interesse einer verbesserten Selbststeuerung der Mitarbeiter*innen sind Führungskräfte angehalten, Entscheidungen zu delegieren, damit die Teammitglieder innen innerhalb ihres Aufgabenspektrums Detailentscheidungen eigenverantwortlich schnell und korrekt treffen können (Vigenschow 2008). Die Übertragung von Entscheidungsbefugnissen an das Team wiederum erfordert ein hohes Maß an Vertrauen ( $\mathrm{Ru}-$ dolph 2017). Schließlich sind Führungskräfte angehalten, auf die persönlichen Bedürfnisse der Mitarbeiter zu achten und deren berufliche Entwicklung zu fördern (Wieczorrek und Mertens 2011).

Um das Selbstmanagement und die Entwicklung der Mitarbeiter*innen aktiv zu fördern, kommt Führungskräften die Rolle eines Coaches zu (Deutsche Gesellschaft für
Projektmanagement und Gessler 2016; Vigenschow 2008). So sollen die Mitarbeiter*innen durch das Coaching lernen, eigene Bedürfnissee besser zu erkennen und mittel- bis langfristig die eigene Karriereentwicklung zu reflektieren und zu planen (Preußig 2015).

Weiterhin wird von Projektleiter*innen erwartet, das Team nicht nur zu führen, sondern im Sinne von Supporting and Cooperating bedarfsabhängig Verhaltensweisen zu zeigen, die denen eines gleichberechtigten Teammitglieds entsprechen. Die Projektleitung sollte in der Lage sein, mit dem gesamten Projektteam kooperativ und respektvoll zum wechselseitigen Vorteil zusammenzuarbeiten und den Austausch innerhalb des Teams zu fördern (vgl. SchmidtRathjens 2007). Eine zentrale Kompetenzdimension bildet die Teamfähigkeit, die es Führungskräften ermöglicht, anpassungsfähig für übergeordnete Ziele zu bleiben, aber gleichzeitig eine kollektive Problemlösung zu ermöglichen, die Ideen aus dem Projektteam berücksichtigt (Preußig 2015). Einfühlungsvermögen und Kooperationsfähigkeit sind von Nöten, um sich in die Mitarbeiter*innen hineinzuversetzen und sie gezielt bei der Erfüllung ihrer Rolle zu unterstützen (Bohinc 2007).

In der Domäne Interacting and Presenting benötigt ein*e Projektleiter*in vor allem eine ausgeprägte Kommunikationsfähigkeit, um im Projektverlauf die interpersonelle Kommunikation mit den Mitarbeiter*innen sowie das Networking mit unterschiedlichen Stakeholdern bedarfsabhängig zu gestalten (Bohinc 2007). So sollten Führungskräfte in der Lage sein, im gesamten Projektteam eine positive Einstellung zum Projekt zu fördern, eine passende Arbeitskultur zu etablieren und zu pflegen und mit allen Mitarbeiter*innen Einzelgespräche als Vorgesetzte*r zu führen (Wieczorrek und Mertens 2011). Nach außen umfasst eine zielgerichtete Kommunikation das Präsentieren von Zwischenständen und Projektergebnissen gegenüber Kunden sowie das Verhandeln von Ressourcen mit den Auftraggebern (Bohinc 2007). Weiterhin sollten Führungskräfte in der Lage sein, konstruktiv mit Konflikten umzugehen und diese zielorientiert zu lösen, da im Projektverlauf Menschen aus verschiedenen Hierarchieebenen und Unternehmensund Interessensbereichen sowie mit unterschiedlichem kulturellen Hintergrund zusammenarbeiten (Wieczorrek und Mertens 2011).

In der Kompetenzdomäne Analyzing and Interpreting stehen wissens- und methodenbezogene Kompetenzen im Vordergrund, welche die Projektleiter*innen befähigen, sachlich-gegenständliche Probleme im Projektverlauf zu durchdringen und selbstorganisiert zu lösen. Um die fachlichen Anliegen der Mitarbeiter*innen zu verstehen, und diese zum eigenverantwortlichen Umgang mit auftretenden Problemen anzuleiten, benötigen Projektleiter*innen ausgeprägtes Fachwissen. Dieses bezieht sich oftmals auf Technologien und/oder Prozesse. Die Anwendung und Ver- 
mittlung fachlicher Expertise bildet eine Voraussetzung, um für das Projektteam die Rolle des Coaches zu übernehmen. Ergänzend wird von Projektleiter*innen erwartet, die benötigten Tools und Instrumente zu beherrschen und selbstständig zu nutzen, was sich im Kontext der Softwareentwicklung vor allem auf die agile Arbeitsweise bezieht (Vigenschow 2008).

Die beiden Kompetenzdomänen Creating and Conceptualizing sowie Adapting and Coping bündeln Verhaltensund Denkmuster, die für den Umgang mit Veränderungen im Projektverlauf relevant sind. Berücksichtigen die Projektleiter*innen diese Veränderungen nicht adäquat, ist der Erfolg des Projektes gefährdet. Creating and Conceptualizing bildet kognitive Kompetenzfacetten im Sinne eines strategischen, antizipierenden Handelns ab, während sich Adapting and Coping auf Kompetenzfacetten zur problemund emotionsbezogenen Bewältigung bezieht. Um einen erfolgreichen Umgang mit Veränderungen zu fördern, übernimmt die Führungskraft die Rolle eines Methoden-Coaches und strategischen Leiters (Deutsche Gesellschaft für Projektmanagement und Gessler 2016; Vigenschow 2008). Die Mitarbeiter*innen sollen befähigt werden, selbst Lösungen für ihre berufsbezogenen Probleme zu finden, da sie über mehr projektspezifisches Wissen als die Führungskraft bzw. Außenstehende verfügen. Zusammenfassend setzt die Rollenübernahme des Coaches somit Kompetenzfacetten in den Bereichen Creating and Conceptualizing, Adapting and Coping sowie Leading and Deciding voraus (vgl. Preußig 2015).

Organizing and Executing bezieht sich im Projektkontext auf planvolles Vorgehen, das die Rahmenbedingungen der Aufgabeerledigung berücksichtigt. Beispielsweise wird von Führungskräften erwartet, das Projektteam mit benötigten Ressourcen wie Wissen, Arbeitsmittelausstattung oder Zeit zu versorgen und diese bedarfsabhängig mit den Auftraggebern zu verhandeln (Wieczorrek und Mertens 2011). Zudem müssen Führungskräfte sicherstellen, dass alle Mitarbeiter*innen über das für ihre Arbeit notwendige Wissen verfügen und bei Bedarf Schulungen initiieren (Wieczorrek und Mertens 2011). Im Kontext der Softwareentwicklung obliegt es Führungskräften zudem, die agile Arbeitsweise auch bei Problemen am Laufen zu halten (Eckkrammer et al. 2010; Wieczorrek und Mertens 2011). Für die Domäne Enterprising and Performing sind keine Kompetenzfacetten benannt, die eine besondere Bedeutung für Projektleiter*innen erlagen.

Zusammenfassend ist bisher kontextübergreifend gut erforscht, mit welchen Kompetenzanforderungen Projektleiter*innen im Allgemeinen konfrontiert sind. Weitgehend unbekannt ist hingegen, welche Kompetenzanforderungen Führungskräfte von agilen Softwareentwicklungsteams aufweisen sollten. Um diese Forschungslücke zu schließen, untersucht die vorliegende Studie mit Hilfe des Great Eight-
Kompetenzrahmens, welche Anforderungen von einer erfolgreichen Projektleitung erwartet werden und wie sich der zugehörige Führungserfolg definieren lässt. Dieses Vorgehen erschien vielversprechend, da bereits mit Hilfe der Great Eight Forschung zu den Kompetenzanforderungen an Mitglieder von Arbeitsgruppen durchgeführt wurde, die Ähnlichkeit mit agil arbeitenden Teams aufweisen. Zeichneten sich die Teams durch kulturell Diversität, hohe Eigenverantwortung, ausgeprägte Entscheidungsfreiheit und virtuelle Kommunikation aus, so kam den Domänen $A d$ apting und Coping und Organizing and Executing (Krumm et al. 2013) sowie Leading and Deciding und Analyzing and Interpreting (Krumm et al. 2016) eine erhöhte Bedeutung zu.

\section{Methode}

\subsection{Stichprobe}

Die Stichprobe setzte sich aus vier Führungskräften verschiedener Ebenen, sechs Projektmitarbeiter*innen (Softwareentwickler oder Consultant) sowie der Assistenz einer Führungskraft eines international operierenden IT-Dienstleisters zusammen. Alle Befragten waren in der Entwicklung und Betreuung interner IT-Systeme tätig. Bei der Auswahl der Stichprobe wurde auf Multiperspektivität geachtet. Sowohl Führungskräfte als auch Mitarbeiter*innen umfassten Personen beiderlei Geschlechts (55\% Männer, $45 \%$ Frauen) aus verschiedenen Projektteams, die sich zudem hinsichtlich ihres Alters (Range: 34 bis 62 Jahre) und der Betriebszugehörigkeit (Range: 3 und 21 Jahre) unterschieden.

\subsection{Vorgehen zur Datenerhebung}

Die Studie wurde im Rahmen einer Bachelorarbeit der Erstautorin durchgeführt. Die o.g. Expert*innen wurden einzeln interviewt. Das Interview wurde als Forschungsmethode ausgewählt, um flexibel und schrittweise möglichst breit Informationen erheben zu können. Die Erstautorin dieser Studie führte alle Interviews mit Hilfe eines semi-strukturierten Leitfadens und einer grafischen Übersicht mit möglichen Kompetenzanforderungen an Führungskräfte durch. Beide Dokumente wurden im Vorfeld der Interviews zusammengestellt, im Rahmen eines Testinterviews erprobt und anschließend überarbeitet.

Der Interviewleitfaden gliederte sich in zwei Teile. Der erste Teil enthielt Fragen zur Arbeitsweise in einem agilen Softwareentwicklungsteam, die als Eisbrecher sowie der gedanklichen Hinführung zum Thema dienten. Sie wurden im Rahmen dieser Studie nicht ausgewertet. Der zweite Teil spezifizierte fünf Fragen zu den Kompetenzanforderun- 
gen an und dem Führungserfolg der zugehörigen Projektleiter*innen (siehe Anhang A). Die Fragen wurden neutral und offen formuliert, um keine Antworten zu suggerieren. Zudem wurde auf eine klare und einfache Sprache geachtet. Entsprechend ihrer Rollen variierte der semi-strukturierte Leitfaden für Mitarbeiter*innen und Führungskräfte leicht. Der Interviewleitfaden für Mitarbeiter*innen umfasst zehn, der für Führungskräfte acht Fragen. Es wurde ein semistrukturiertes Format gewählt, da ein solcher Leitfaden dem Interviewer Flexibilität bietet, die Reihenfolge und Formulierung der Fragen abhängig von Gesprächsverlauf und Interviewpartner zu variieren. Gleichzeitig sorgt er dafür, dass interviewübergreifend die gleichen Informationen vollständig erhoben werden (Gläser und Laudel 2010).

Die Kompetenzübersicht enthielt Anforderungen, die an Projektleiter*innen im Allgemeinen gestellt werden (Bohinc 2007; Deutsche Gesellschaft für Projektmanagement und Gessler 2016; Kuster et al. 2006). Die Übersicht wurde unter Zuhilfenahme des im deutschsprachigen Raum gebräuchlichen vierdimensionalen Kompetenzmodells (Blickle 2019; Schmidt-Rathjens 2007; Sonntag und Schaper 2006) erstellt, mit dem die befragte Zielgruppe vertraut war und welches die Kompetenzdimensionen der Great Eight weitgehend widerspiegelte. Falls die Befragten die gestellten Fragen nicht beantworten konnten, diente diese Übersicht als Hilfsmittel, um die Wichtigkeit der gelisteten Einzelkompetenzen im Interview zu überprüfen (Anhang B). Für die Fachkompetenz, die im Great EightModell der Kompetenzdomäne Analyzing and Interpreting entspricht, wurden keine Beispiele gegeben, da diese Aspekte durch die Interviews genauer erschlossen werden sollten.

Alle Interviews folgten demselben Ablauf. Zu Beginn stellte sich die Interviewerin vor, erläuterte das Interviewziel und wies auf die Anonymität der erhobenen Daten hin. Alle Expert*innen erteilten schriftlich ihre Einwilligung, das Interview digital aufzeichnen und die erhobenen Daten auswerten zu lassen. Stellte sich während des Interviews heraus, dass eine Frage für einen Befragten unzutreffend oder kritisch war, wurde diese nicht gestellt. Am Ende des Interviews wurde jedem Interviewten die Möglichkeit eingeräumt, wichtige Aspekte ergänzen, die im Verlauf des Interviews bisher keine Berücksichtigung gefunden hatten.

Die Interviews wurden über einen Zeitraum von rund drei Wochen durchgeführt. Pro Tag fanden ein bis drei Interviews statt. Die Dauer betrug durchschnittlich 33 min. Zehn von elf Interviewten wurden auf Deutsch befragt. Ein russischsprachiger Interviewpartner wurde auf Englisch befragt. Alle Interviews wurden mit einem Diktiergerät aufgezeichnet, zusätzlich von Hand protokolliert und zeitnah von der Interviewerin selbst transkribiert, um die Gesprächsinhalte so exakt wie möglich abzubilden. Hierfür wurde das gesprochene Wort in einer Worddatei verschriftet. Nach jedem Interview verfasste die Interviewerin einen kurzen Bericht, in dem die Stimmung im Interview, die Haltung des Befragten gegenüber der Interviewerin sowie sonstige Besonderheiten festgehalten wurden. Zur Transkription wurden der Interviewbericht und das Protokoll ergänzend herangezogen.

\subsection{Auswertung der Interviews}

Die anonymisierten Transkripte wurden mittels qualitativer Inhaltsanalyse nach Gläser und Laudel (2010) anhand eines Kategoriensystems mit Hilfe der Software MAXQDA computergestützt ausgewertet. Bei diesem Verfahren besteht die Möglichkeit, komplexe Bedeutungsgefüge aus den Transkripten zu extrahieren und während des gesamten Auswertungsprozesses offen für unvorhergesehene Informationen zu bleiben (Kuster et al. 2006). Auf Basis theoretischer Vorüberlegungen wurde ein Suchraster in Form von Kategorien erstellt, anhand dessen relevante Informationen aus den Transkripten extrahiert wurden. Es wurden fünf Hauptkategorien gebildet, die bei Bedarf während der Extraktion ergänzt und angepasst werden konnten.

Die erste Kategorie umfasste Aussagen zu den Aufgaben von Führungskräften, die den Kompetenzfacetten im Modell der Great Eight entsprachen. Die zweite Kategorie bündelte die Kompetenzdimensionen im Sinne der Great Eight. Zudem wurde eine dritte Kategorie zur Rolle von Führungskräften als Vertrauenspersonen und eine vierte zur Rolle von Führungskräften als Vorbild angelegt, da von den Befragten häufig Aussagen zu diesen Aspekten getroffen wurden. In der fünften Kategorie wurden Nennungen zum Führungserfolg zusammengefasst.

Die Transkripte wurden in MAXQDA eingelesen und die o.g. Kategorien zum Codieren angelegt. Nacheinander wurden in den Transkripten Textpassagen markiert und der/den entsprechenden Kategorie(n) zugeordnet. Für jede Kategorie wurde anschließend eine Übersicht der Transkriptteile erstellt und als Tabelle exportiert. Um die Qualität der Daten zu verbessern, wurden verstreute aber semantisch ähnliche Informationen zusammengefasst und mögliche Fehler korrigiert (Gläser und Laudel 2010). Dazu wurden die Extraktionstabellen kopiert und Informationen im Text, die zusammengehören, in der gleichen Farbe markiert. Die auf diese Weise aufbereiteten und extrahierten Transkriptstücke wurden im letzten Schritt mit theoretischen Vorüberlegungen zu hierarchisch genesteten Kompetenzanforderungen im Sinne des Great Eight-Rahmenmodells in Verbindung gebracht und interpretiert. Ergänzend wurden die Häufigkeiten der kategorisierten Anforderungen analysiert. 
Tab. 2 Ermittelte Kompetenzanforderungen, differenziert nach -dimensionen und -facetten, systematisiert anhand des Great EightKompetenzrahmens

\begin{tabular}{|c|c|c|}
\hline $\begin{array}{l}\text { Great Eight- } \\
\text { Domäne }\end{array}$ & Kompetenzdimensionen & Kompetenzfacetten auf der Verhaltensebene \\
\hline \multirow{4}{*}{$\begin{array}{l}\text { Leading and } \\
\text { Deciding }\end{array}$} & \multirow{4}{*}{$\begin{array}{l}\text { Motivationsfähigkeit } \\
\text { Mitarbeiterförderung }\end{array}$} & Gibt regelmäßig Feedback (providing direction and coordinating action) \\
\hline & & Mitarbeiter*innen motivieren und loben (Motivating others) \\
\hline & & Coaching von Mitarbeiter*innen (Coaching) \\
\hline & & Überwachen, dass agile Arbeitsweise eingehalten wird (Supervising and Monitoring) \\
\hline \multirow[t]{5}{*}{$\begin{array}{l}\text { Supporting und } \\
\text { Cooperating }\end{array}$} & \multirow[t]{5}{*}{ Teamfähigkeit } & $\begin{array}{l}\text { Team bei Zielbildung, Aufgabenerledigung, Lösungsfindung unterstützen (Supporting } \\
\text { others) }\end{array}$ \\
\hline & & $\begin{array}{l}\text { Bei Bedarf als Schutzschild für die Mitarbeiter*innen fungieren (Showing social responsibi- } \\
\text { lity) }\end{array}$ \\
\hline & & Team zur Zusammenarbeit ermutigen (Building team spirit) \\
\hline & & Kommunikation im Team fördern, Meetings moderieren (Communicating proactively) \\
\hline & & $\begin{array}{l}\text { Mitarbeiter*innen mit Rat und Wissen zum agilen Arbeiten zur Seite stehen (Consulting } \\
\text { others) }\end{array}$ \\
\hline \multirow[t]{2}{*}{$\begin{array}{l}\text { Interacting and } \\
\text { Presenting }\end{array}$} & Kommunikationsfähigkeit & $\begin{array}{l}\text { Mitarbeitergespräche führen (Shaping conversation) } \\
\text { Agile Werte vermitteln und vorleben, im Team etablieren (Explaining concepts and opinions) }\end{array}$ \\
\hline & Konfliktfähigkeit & Proaktiver Umgang von Konflikten (Managing conflict) \\
\hline $\begin{array}{l}\text { Analyzing and } \\
\text { Interpreting }\end{array}$ & $\begin{array}{l}\text { Kenntnisse IT-Branche } \\
\text { Überblickswissen Soft- } \\
\text { wareentwicklung } \\
\text { Agile Arbeitsmethoden }\end{array}$ & $\begin{array}{l}\text { Anwendung und Weitergabe des Fachwissens im Bereich IT und Softwareentwicklung (App- } \\
\text { lying/sharing technical expertise) } \\
\text { Anwendung und Weitergabe des Wissens zur agilen Arbeitsweise (Applying/sharing technical } \\
\text { expertise) }\end{array}$ \\
\hline $\begin{array}{l}\text { Creating and } \\
\text { Conceptualizing }\end{array}$ & Offenheit & $\begin{array}{l}\text { Verbesserungen des agilen Arbeitens im Team anregen (Encouraging organizational learn- } \\
\text { ing) }\end{array}$ \\
\hline \multirow{3}{*}{$\begin{array}{l}\text { Organizing and } \\
\text { Executing }\end{array}$} & \multirow{3}{*}{$\begin{array}{l}\text { Zeit- und } \\
\text { Ressourcenmanagement }\end{array}$} & Zeitmanagement des agilen Arbeitens im Team (Managing time) \\
\hline & & $\begin{array}{l}\text { Planung und Sicherstellung von Ressourcen für das Team in Bezug auf Wissen, Arbeitsmit- } \\
\text { tel, räumliche Zusammenarbeit (Managing resources) }\end{array}$ \\
\hline & & $\begin{array}{l}\text { Planung und Management externer Ressourcen, u. a. Akquise und Abrechnung externer } \\
\text { Mitarbeiter*innen (Managing resources) }\end{array}$ \\
\hline $\begin{array}{l}\text { Adapting and } \\
\text { Coping }\end{array}$ & $\begin{array}{l}\text { Proaktiver Umgang mit } \\
\text { Fehlern }\end{array}$ & Probleme in Arbeitsabläufen erkennen/beheben (Handling criticism; coping with pressure) \\
\hline $\begin{array}{l}\text { Enterprising and } \\
\text { Performing }\end{array}$ & $\begin{array}{l}\text { Begeisterungsfähigkeit } \\
\text { für Softwareentwicklung }\end{array}$ & $\begin{array}{l}\text { Begeisterungsfähigkeit für Softwareentwicklung, um bei Rückschlägen eine erfolgreiche } \\
\text { Implementierung sicherzustellen (Working energetically and enthusiastically) }\end{array}$ \\
\hline
\end{tabular}

Kompetenzdimensionen und -facetten, die spezifisch für den Kontext der agilen Softwareentwicklung sind, sind kursiv gesetzt; für die identifizierten Kompetenzfacetten sind in Klammern die äquivalenten englischen Bezeichnungen nach Bartram (2005) angegeben

\section{Ergebnisse}

Für Führungskräfte von agilen Softwareentwicklerteams konnte ein Anforderungsprofil mit 20 Kompetenzfacetten und 12 übergeordneten Kompetenzdimensionen identifiziert werden, die sich wiederum nach den acht Domänen der Great Eight nach Bartram (2005) clustern ließen. Die Ergebnisse sind in Tab. 2 dargestellt.

Die 20 Kompetenzfacetten bilden relevante Verhaltensweisen ab, die Projektleiter*innen im Führungsalltag zeigen sollten, um ein Team von Softwareentwickler*innen erfolgreich zu leiten. Jede der 20 Nennungen korrespondierte direkt mit einer englischsprachigen Kompetenzfacette nach Bartram (2005), was für die Validität der Befunde spricht (für Details siehe Tab. 2). Weiterhin ließen sich eine bis drei Kompetenzfacetten jeweils einer der 12 übergeordneten Kompetenzdimensionen zuordnen, wobei jede Domäne der Great Eight durch mindestens eine Kompetenzdimen- sion repräsentiert war. In Anlehnung an die Prinzipien des agilen Arbeitens (Beck et al. 2001) liegt Führungserfolg vor, wenn die Mitarbeiter*innen gut zusammenarbeiten, sodass lauffähige Software ressourceneffizient nach Kundenwünschen entwickelt wird und gleichzeitig die Teammitglieder eine hohe Arbeitszufriedenheit berichten.

Im Vergleich zu klassischen Führungskräften - und in Übereinstimmung mit bisheriger Forschung zu den Kompetenzanforderungen bei Projektleiter*innen - kommt den Domänen Leading and Deciding, Supporting and Cooperating, Interacting and Presenting sowie Analyzing and Interpreting für die Leitung eines Entwicklungsteams eine erhöhte Bedeutung zu (Bohinc 2007; Deutsche Gesellschaft für Projektmanagement und Gessler 2016; Geipel 2003; Vigenschow 2008). Drei Viertel aller Kompetenzfacetten und zwei Drittel der identifizierten Kompetenzdimensionen konzentrierten sich auf diese vier Kompetenzdomänen. Ein besonders hohes Augenmerk lag wiederum auf ent- 
wicklungs- und förderorientierten Kompetenzfacetten der Domänen Leading and Deciding sowie Supporting and Cooperating, die knapp die Hälfte aller Nennungen ausmachten.

\subsection{Leading and Deciding}

Die Domäne Leading and Deciding umfasst führungsbezogene Verhaltensweisen, die sich auf die Kompetenzdimensionen Motivationsfähigkeit und Mitarbeiterförderung konzentrieren. Von den Führungskräften wird erwartet, ihre Mitarbeiter*innen regelmäßig zu motivieren und zu loben, was nach Wieczorrek und Mertens (2011) voraussetzt, projektbezogene Ziele und Visionen klar zu vermitteln, die im Projektteam anfallenden Aufgaben zu planen und deren Erledigung zu überwachen. Im Sinne relevanter Kompetenzfacetten sind Führungskräfte verantwortlich, den Mitarbeiter*innen regelmäßig Feedback zum Vorgehen und zur Qualität geben und die Einhaltung der agilen Arbeitsweise zu überwachen. Eine hohe Motivationsfähigkeit ist zudem nötig, um Mitarbeiter*innen zu animieren, mehr Verantwortung zu übernehmen und sich Produktanforderungen aktiv anzueignen.

Weiterhin sind Projektleiter*innen dafür zuständig, die Mitarbeiter*innen bedarfsabhängig zu coachen. Durch die hohe Entscheidungsfreiheit und eigenständige Arbeitsweise kann das Team nur dann erfolgreich arbeiten, wenn alle Mitglieder eine hohe Leistung erbringen. Sind die Mitarbeiter*innen (noch) nicht bereit, sich bestimmte Aufgaben zuzutrauen und Verantwortung zu übernehmen, sind Führungskräfte angehalten, sie im Sinne einer Persönlichkeitsentwicklung dazu anregen. Dazu ist eine Bereitschaft und Befähigung zur Mitarbeiterförderung wichtig, welche Projektleiter*innen in der Lage versetzt, die Stärken der Teammitglieder innen zu erkennen und deren berufliche Entwicklung gezielt zu fördern (Wieczorrek und Mertens 2011). Sind die genannten Kompetenzfacetten hoch ausgeprägt, befähigen sie Führungskräfte, bei Bedarf für die Mitarbeiter*innen die Rolle eines Coaches zu übernehmen.

\subsection{Supporting and Cooperating}

Die Kompetenzdomäne Supporting and Cooperating wird durch die Kompetenzdimension Teamfähigkeit repräsentiert, die Verhaltensweisen bündelt, die denen eines gleichberechtigten Teammitglieds entsprechen. Führungskräfte sollten die Mitarbeiter*innen bei der Zielbildung, Aufgabeerledigung und Lösungsfindung unterstützen und im Hinblick auf die agile Arbeitsweise beraten, die teambezogene Zusammenarbeit und den Austausch fördern und bei Bedarf als Schutzschild für die Teammitglieder nach außen fungieren.

\subsection{Interacting and Presenting}

Die beiden Kompetenzfacetten Kommunikationsfähigkeit und Konfliktfähigkeit bilden die konstituierenden Kompetenzdimensionen der Domäne Interacting and Presenting. Im Sinne relevanter Kompetenzfacetten sollten Projektleiter*innen in der Lage sein, zielgruppengerecht und anlassbezogen zu kommunizieren, was das Führen von Mitarbeitergesprächen sowie das Vorleben und Vermitteln agiler Werte einschließt. Schaffen es Führungskräfte nicht, die agilen Werte im Team zu etablieren, so gefährdet dies den Projekterfolg, da die agile Arbeitsmethode einen ständigen Informationsaustausch aller Beteiligten voraussetzt. Eine ausgeprägte Konfliktlösungsfähigkeit ist wichtig, da durch den ständigen Austausch und die häufigen Veränderungen ein erhöhtes Konfliktpotenzial im Arbeitsalltag besteht. Die Führungskräfte sollten beispielsweise in der Lage sein, die Mitarbeiter*innen im Rahmen einer Mediation dabei zu unterstützen, ihre Konflikte selbstständig zu lösen.

\subsection{Analyzing and Interpreting}

Die Kompetenzdomäne Analyzing and Interpreting bündelt Fach- und Methodenwissen, welches die Projektleiter*innen befähigt, fachliche Probleme im Projektverlauf $\mathrm{zu}$ analysieren und $\mathrm{zu}$ verstehen. Die wichtigste Anforderung in diesem Bereich stellt IT-branchenspezifisches Hintergrundwissen dar, um den Projektfortschritt fachlich $\mathrm{zu}$ unterstützen und die Anliegen der Mitarbeiter*innen verstehen und lösen zu können. Überblickswissen im Bereich Softwareentwicklung wurde als zweitwichtigste Kompetenzanforderung identifiziert. Verfügen Führungskräfte über die entsprechende Expertise, wird bei Projektbeteiligten Vertrauen und eine höhere Akzeptanz getroffener Entscheidungen begünstigt. Weiterhin ist wichtig, dass die Führungskräfte über Wissen zu agilen Werten und Methoden sowie deren korrekter Anwendung und Vermittlung verfügen. Dem letztgenannte Kompetenzdimension spielt eine vergleichsweise untergeordnete Rolle, wenn die Teammitglieder bereits über Erfahrung mit agilen Arbeitsmethoden verfügen.

\subsection{Creating and Conceptualizing sowie Adapting and Coping}

Die beiden Kompetenzdomänen Creating and Conceptualizing sowie Adapting and Coping repräsentieren Verhaltensund Denkmuster, die es den Führungskräften ermöglichen, adäquat mit auftretenden Problemen im Projektverlauf umzugehen, was ein wichtiges Merkmal der agilen Arbeitsweise bildet. Um schnell und strategisch auf Veränderungen zu reagieren, Verbesserungen im agilen Arbeiten anzuregen und zugehörige Lernprozesse bei den Mitarbeiter*innen an- 
zustoßen, sollten Projektleiter*innen im Bereich Creating and Conceptualizing über eine hohe Offenheit verfügen. Der proaktive und professionelle Umgang mit Fehlern bildet die zentrale Anforderung im Bereich Adapting and Coping, da die Mitglieder in agilen Teams selbstorganisiert zusammenarbeiten und eigenverantwortlich neue Dinge ausprobieren, wodurch Innovation möglich wird, aber die Fehlerwahrscheinlichkeit steigt. Aus diesem Grund sollten Führungskräfte mit (eigenen) Fehlern im Team konstruktiv umgehen, die Mitarbeiter*innen dazu ermutigen, sich auszuprobieren und ihnen die Angst vor Fehlern nehmen. Bringen Führungskräfte zudem eine hohe Offenheit mit, können sie eine offene Herangehensweise im Sinne agiler Methoden authentischer an ihre Mitarbeiter*innen vermitteln.

\subsection{Organizing and Executing}

Die Domäne Organizing and Executing wird durch die Kompetenzdimension Zeit- und Ressourcenmanagement abgebildet, was Projektleiter*innen ein planvolles Vorgehen zur Aufgabenerledigung ermöglicht. Die Führungskräfte stehen vor der Herausforderung, den Projektmitgliedern kontinuierlich gute Rahmenbedingungen für ihre Arbeit zu schaffen. Dies umfasst eine Versorgung mit Wissen und Arbeitsmitteln sowie eine Sicherstellung der räumlichen Zusammenarbeit. Ferner sind die Führungskräfte verantwortlich, externe Personalressourcen $\mathrm{zu}$ planen und $\mathrm{zu}$ verwalten, was sich primär auf die Akquise und Integration von externen Spezialist*innen zur Verstärkung des Teams bezieht. Weiterhin ist ein adäquates Zeitmanagement relevant. Begründet wird dies mit häufigen Besprechungen wie täglichen Daily-Standup-Meetings, Sprintplanungen und Retrospektiven. Der rege Austausch ermöglicht den Erfolg der agilen Arbeitsweise. Werden Meeting-Zeiten jedoch überschritten oder nicht auf die Einhaltung von Redeanteilen geachtet, leidet die Produktivität. Ein gutes Zeitmanagement von Führungskräften wirkt vorbeugend und erleichtert Sprintplanungen und eine korrekte Abschätzung der Auslastung im Team.

\subsection{Enterprising and Performing}

Im Bereich Enterprising and Performing ist es schließlich wichtig, dass sich Führungskräfte für das zu entwickelnde Produkt begeistern können, um bei Problemen den Entwicklungsprozess bis zur Implementierung der Software durchzuhalten. Gerade im agilen Arbeitsumfeld geht es darum, das Produkt schrittweise gemeinsam mit dem Kunden $\mathrm{zu}$ optimieren, was langwierig und austauschintensiv sein kann. Für das Implementieren neuer Features und das Umsetzen von Anforderungen sind somit Begeisterung für die Softwareentwicklung nötig.

\section{Fazit und Transfer}

In der vorliegenden Studie wurden Kompetenzanforderungen an und der Führungserfolg von Projektleiter*innen im agilen, hochdigitalisierten Arbeitsumfeld der Softwareentwicklung untersucht und in den Kompetenzrahmen der Great Eight einsortiert. Die Anforderungen in den Domänen Analyzing and Interpreting sowie Enterprising and Performing sind spezifisch für den Arbeitskontext der agilen Softwareentwicklung und unterscheiden sich von denen sonstiger Projektleitungen. Ähnlichkeiten zeigen sich hingegen bei der hohen Bedeutung der Domänen Leading and Deciding, Supporting and Cooperating sowie Interacting and Presenting. Die zugehörigen Kompetenzfacetten fokussieren im Sinne geforderter Verhaltensweisen auf eine individualisierte Unterstützung des Entwicklerteams bei der selbstverantwortlichen Erledigung der Aufgaben, eine Förderung der teaminternen Kommunikation und Zusammenarbeit sowie den Umgang mit Veränderungen beim agilen Arbeiten. Entsprechend dieses Portfolios kommt der Projektleitung die Rolle eines Coachs für die fachliche, karrierebezogene und persönliche Entwicklung der Mitarbeiter*innen zu. Bei örtlich verstreuten Teammitgliedern ergibt sich ein Bedarf an schnellen und flexibel realisierbaren Schulungsmethoden. In der Praxis bietet sich dafür digital gestütztes, berufsbegleitendes Lernen an, welches auf den Methoden des agilen Arbeitens basiert, und den Projektmitgliedern ermöglicht, bedarfsabhängig aber örtlich unabhängig Wissen und Problemlösekompetenzen aufzubauen (Höhne et al. 2017).

Zusammenfassend wird von Projektleiter*innen in der Softwareentwicklung eine Kombination aus tätigkeitsfeldspezifischen und kontextunabhängigen Kompetenzanforderungen erwartet. Wir empfehlen weitere Forschung, um die identifizierten Kompetenzanforderungen mit bestehenden Rahmenmodellen (Deutsche Gesellschaft für Personalführung 2016; European Committee for Standardization (CEN) 2016; Kluzer und Pujol Priego 2018) abzugleichen, um theoretische und praxisgeleitete Ansätze in einem Kompetenzmodell für Führungskräfte in digitalisierten Arbeitsumgebungen zu bündeln.

Die vorliegende Studie schließt eine Forschungslücke, da die kombinierte Identifikation von Erfolgskriterien und Kompetenzanforderungen auf der Ebene von Domänen, Dimensionen und Verhaltensfacetten eine Voraussetzung darstellt, um geeignete Bewerber*innen für eine Führungsposition in einem agil arbeitenden Softwareentwicklungsteam anforderungsbasiert zu bestimmen. Eine kompetenzbasierte Personalauswahl und -entwicklung bietet mehrere Vorteile (Blickle 2019; Schmidt-Rathjens 2007; Sonntag und Schmidt-Rathjens 2005). Dem Unternehmen hilft sie, Entscheidungen im Auswahlprozess kriteriengestützt zu optimieren, um das Risiko für Fehlbesetzungen oder 
subjektive Präferenzen der Auswahlkommission zu verringern. Den ausgewählten Kandidat*innen hilft sie, anstehende Führungsaufgaben erfolgreich zu meistern und einen größeren Berufserfolg zu erzielen (Zühlke 2017). Auch für Unternehmen, die auf agile Arbeitsmethoden umstellen, beinhaltet die vorliegende Studie wichtige Hinweise, um Führungskräfte zielgerichtet einzusetzen.

Funding Open Access funding enabled and organized by Projekt DEAL.

\section{Anhang}

\section{Anhang A}

\section{Interviewleitfaden}

Anmerkung: Bei dem Interviewleitfaden für Führungskräfte entfallen Frage 3 und 4 im zweiten Teil.

\section{Interviewleitfaden für Mitarbeiter}

Interviewpartner:

Bereich/Team:

Position:

Datum des Interviews:

Dauer des Interviews:

\section{Vorbemerkung}

Kurzvorstellung Interviewer Vielen Dank, dass Sie sich Zeit für das Interview genommen haben. Das Ziel dieser Untersuchung besteht darin herauszufinden, welche Kompetenzanforderungen an Führungskräfte in agilen Softwareentwicklerteams bestehen. Das Ziel des Interviews ist es, wichtige Kompetenzen zu identifizieren. Das Interview besteht aus zehn offenen Fragen und dauert ca. 30-45 min. Zuerst stelle ich fünf allgemeine Fragen zur Arbeitsweise von agilen Softwareentwicklerteams, anschließend fünf Fragen zur Führungskraft und deren Kompetenzen. Alles, was Sie mir erzählen wird vertraulich behandelt. Falls später etwas darüber geschrieben wird, werden die Ergebnisse anonymisiert und es sind keine Rückschlüsse auf die Person möglich. Besteht Einverständnis mit einer Tonbandaufnahme (um den exakten Wortlaut zu erfassen, zur Datensicherung, als Hilfe zur Datenauswertung, da beim Protokollieren wichtige Details verloren gehen können)?
Allgemeine Fragen zur Arbeit in einem agilen SoftwareEntwicklerteam:

1. In dieser Untersuchung geht es um Führungskräfte in agilen Softwareentwicklerteams. Seit wann arbeitet ihr Team nach agilen Methoden?

- Seit wann arbeiten Sie als Mitglied des Teams nach agilen Methoden?

2. Was verstehen Sie unter einer agilen Arbeitsweise und wie wird diese im Arbeitsalltag umgesetzt?

- Typische Merkmale: Tägliche „Daily-Standup-Meetings", Scrum?

3. Was läuft gut bei der agilen Arbeitsweise?

- Im Vergleich zu vorher (nach klassischer Arbeitsweise/Wasserfallmodell)?

4. Was läuft nicht gut bei der agilen Arbeitsweise?

- Im Vergleich zu vorher (nach klassischer Arbeitsweise/Wasserfallmodell)?

5. Welche Herausforderungen stellt die agile Arbeitsweise im Berufsalltag an das Softwareentwicklerteam/einzelne Teammitglieder?

- Bsp.: schwierige Zeitplanung, ungewisse Budgetplanung, kurzfristige Kundenanforderungen, hoher Zeitdruck

\section{Fragen zu den Kompetenzen der Führungskraft:}

1. Welche Hauptaufgaben hat die Führungskraft eines agilen Softwareentwicklerteams?

2. Welche Kompetenzen benötigt die Führungskraft eines agilen Softwareentwicklerteams?

- Abbildung dazu: Für jede Kompetenzkategorie wichtige auswählen (3-5) und gewichten

- Welche Rolle spielt die Sozialkompetenz? Welche besonders und warum?

- Welche Rolle spielt die Selbstkompetenz? Welche besonders und warum?

- Welche Rolle spielt die Methodenkompetenz? Welche besonders und warum?

- Welche Rolle spielt die Fachkompetenz? Und warum?

3. Welche Eigenschaften sind Ihnen persönlich bei Ihrer (direkten) Führungskraft besonders wichtig?

- Egal aus welcher Kompetenzkategorie

- Was schätzen Sie an Ihrer Führungskraft?

4. Sie hatten gesagt, dass als

Herausforderungen im Berufsalltag auftreten. Was hat Ihre Führungskraft getan um in solchen Situationen zu helfen?

- War das hilfreich?

- Was hätte die Führungskraft alternativ tun sollen? 
5. Woran machen Sie den Führungserfolg in einem agilen

Softwareentwicklerteam fest?

- Hohe Kundenorientierung $\rightarrow$ zufriedene Kunden, hoher Umsatz bzw. Gewinn $\rightarrow$ Unternehmenserfolg, hohe Zielerfüllungsquote im Team $\rightarrow$ zufriedene Mitarbeiter?

Das waren alle Fragen. Gibt es weitere wichtige Aspekte zum Thema, die Sie mir mitteilen wollen und die bisher im Interview keine Berücksichtigung gefunden haben?

Vielen Dank.

\section{Anhang B}

\section{Übersicht Kompetenzanforderungen}

\begin{tabular}{|c|c|c|c|}
\hline Sozialkompetenz & Selbstkompetenz & Methodenkompetenz & Fachkompetenz \\
\hline $\begin{array}{l}\text { Fähigkeit, kooperativ und } \\
\text { kommunikativ selbstorganisiert } \\
\text { zu handeln }\end{array}$ & $\begin{array}{l}\text { Fähigkeit, sich realistisch } \\
\text { einzuschätzen und } \\
\text { weiterzuentwickeln }\end{array}$ & $\begin{array}{l}\text { kognitive Fähigkeit, die einer } \\
\text { Person helfen Aufgaben } \\
\text { selbstständig zu bearbeiten }\end{array}$ & $\begin{array}{l}\text { Fähigkeit, sachlich- } \\
\text { gegenständliche Probleme } \\
\text { geistig und physisch } \\
\text { selbstorganisiert anzugehen }\end{array}$ \\
\hline $\begin{array}{l}\text { - Kommunikationsfähigkeit } \\
\text { - Feedback } \\
\text { - Kesprächsführung } \\
\text { - Konfliktlösungsfähigkeit } \\
\text { - Kooperationsbereitschaft } \\
\text { - } \text { Metivationsfähigkeit } \\
\text { - } \text { Mitarbeiterförderung } \\
\text { - Teamfähigkeit } \\
\text { - Kritikfähigkeit } \\
\text { - Kompromissfähigkeit } \\
\text { - } \text { Frustrationstoleranz } \\
\text { - } \text { Durchsetzungsvermögen }\end{array}$ & $\begin{array}{l}\text { - Selbstwahrnehmung } \\
\text { - Selbstbewusstsein } \\
\text { - } \text { Umgang mit Fehlern } \\
\text { - Sellexionsfähigkeit } \\
\text { - Offenheit für Veränderung } \\
\text { - Ehrgeiz } \\
\text { - } \text { Begeisterungsfähigkeit } \\
\text { - } \text { Zeurteilungsvermögen } \\
\text { - } \text { Belastroritäten } \\
\text { - Verständnisfähigkeit } \\
\text { - Emotionsregulation } \\
\text { - Stressbewältigung }\end{array}$ & $\begin{array}{l}\text { - Analysefähigkeit } \\
\text { - Entscheidungsfähigkeit } \\
\text { - } \text { Arbeitsplanung } \\
\text { - Strukturierungsfähigkeit } \\
\text { - Zeitmanagement } \\
\text { - Problemlösungstechniken }\end{array}$ & $\begin{array}{l}\text { - Fachkenntnisse } \\
\text { - Fachsprache } \\
\text { - Erwerb, Verstehen und } \\
\text { Bewertung von Wissen }\end{array}$ \\
\hline
\end{tabular}

Open Access Dieser Artikel wird unter der Creative Commons Namensnennung 4.0 International Lizenz veröffentlicht, welche die Nutzung, Vervielfältigung, Bearbeitung, Verbreitung und Wiedergabe in jeglichem Medium und Format erlaubt, sofern Sie den/die ursprünglichen Autor(en) und die Quelle ordnungsgemäß nennen, einen Link zur Creative Commons Lizenz beifügen und angeben, ob Änderungen vorgenommen wurden.

Die in diesem Artikel enthaltenen Bilder und sonstiges Drittmaterial unterliegen ebenfalls der genannten Creative Commons Lizenz, sofern sich aus der Abbildungslegende nichts anderes ergibt. Sofern das betreffende Material nicht unter der genannten Creative Commons Lizenz steht und die betreffende Handlung nicht nach gesetzlichen Vorschriften erlaubt ist, ist für die oben aufgeführten Weiterverwendungen des Materials die Einwilligung des jeweiligen Rechteinhabers einzuholen.

Weitere Details zur Lizenz entnehmen Sie bitte der Lizenzinformation auf http://creativecommons.org/licenses/by/4.0/deed.de.

\section{Literatur}

Bartram, D. (2005). The great eight competencies: a criterion-centric approach to validation. Journal of Applied Psychology, 90, 1185-1203.

Beck, K., Beedle, M., van Bennekum, A., Cockburn, A., Cunningham, W., et al. (2001). Manifesto for agile software development. https://agilemanifesto.org/. Zugegriffen: 15. Mai 2020.

Blickle, G. (2019). Anforderungsanalyse. In F. W. Nerdinger, G. Blickle \& N. Schaper (Hrsg.), Arbeits- und Organisationspsychologie (S. 235-249). Berlin: Springer.

Bohinc, T. (2007). Projektmanagement. Soft Skills für Projektleiter. Offenbach: GABAL.

Deutsche Gesellschaft für Personalführung (2016). Leitfaden: Kompetenzen in digitalisierten Unternehmen. https://www.dgfp.de/ fileadmin/user_upload/DGFP_e.V/Medien/Publikationen/Praxis papiere/201602_Praxispapier_Kompentenzen-im-digitalisiertenUnternehmen.pdf. Zugegriffen: 22. Mai 2020.

Deutsche Gesellschaft für Projektmanagement e. V., \& Gessler, M. (Hrsg.). (2016). Kompetenzbasiertes Projektmanagement (PM3). Handbuch für die Projektarbeit, Qualifizierung und Zertifizierung. Nürnberg: Deutsche Gesellschaft für Projektmanagement e. V.. 
Eckkrammer, T., Eckkrammer, F., \& Gollner, H. (2010). Agiles ITProjektmanagement im Überblick. In E. Tiemeyer (Hrsg.), Handbuch IT-Projektmanagement. Vorgehensmodelle, Managementinstrumente, Good Practices (S. 73-112). München: Hanser.

European Committee for Standardization (CEN) (2016). European e-competence framework 3.0. https://www.ecompetences.eu/de/. Zugegriffen: 30. Juni 2020.

Geipel, P. (2003). Der IT-Projektmanager: Arbeitstechniken, Checklisten und soziale Kompetenz. München: Addison-Wesley.

Gläser, J., \& Laudel, G. (2010). Experteninterviews und qualitative Inhaltsanalyse. Wiesbaden: VS.

Gloger, B., \& Häusling, A. (2011). Erfolgreich mit Scrum - Einflussfaktor Personalmanagement. Finden und Binden von Mitarbeitern in agilen Unternehmen. München: Hanser.

Höhne, B.P., Bräutigam, S., Longmuß, J., \& Schindler, F. (2017). Agiles Lernen am Arbeitsplatz - eine neue Lernkultur in Zeiten der Digitalisierung. Zeitschrift für Arbeitswissenschaft, 71, 110-119.

Kluzer, S., \& Pujol Priego, L. (2018). DigComp into Action-Get inspired, make it happen. In S. Carretero, Y. Punie, R. Vuorikari, M. Cabrera \& W. O'Keefe (Hrsg.), JRC Science for Policy Report. EUR 29115 EN. Luxembourg: Publications Office of the European Union. https://publications.jrc.ec.europa.eu/ repository/bitstream/JRC110624/dc_guide_may18.pdf. Gesehen 23.11.2019.

Krumm, S., Kanthak, J., Hartmann, K., \& Hertel, G. (2016). What does it take to be a virtual team player? The knowledge, skills, abilities, and other characteristics required in virtual teams. Human Performance, 29, 123-142. https://doi.org/10.1080/08959285.2016. 1154061.

Krumm, S., Terwiel, K., \& Hertel, G. (2013). Challenges in norm formation and adherence: the knowledge, skills, and ability requirements of virtual and traditional cross-cultural teams. Journal of Personnel Psychology, 12, 33-44. https://doi.org/10.1027/18665888/a000077.

Kuster, J., Huber, E., Lippmann, R., Schmid, A., Schneider, E., Witschi, U., \& Wüst, R. (2006). Handbuch Projektmanagement. Berlin: Springer.

Onpulson (2018). Agilität. http://www.onpulson.de/lexikon/agilitaet/. Zugegriffen: 13. Febr. 2018.

Preußig, J. (2015). Agiles Projektmanagement: Scrum, Use Cases, Task Boards \& Co. Freiburg: Haufe.

Rudolph, A. (2017). Agile Organisation. Arbeit und Arbeitsrecht, 3, 165-167.

Schmidt-Rathjens, C. (2007). Anforderungsanalyse und Kompetenzmodellierung. In H. Schuler \& K.-H. Sonntag (Hrsg.), Handbuch der Arbeits- und Organisationspsychologie. Handbuch der Psychologie, (Bd. 6, S. 592-601). Göttingen: Hogrefe.

Sonntag, K., \& Schmidt-Rathjens, C. (2005). Anforderungsanalyse und Kompetenzmodelle. In P. Gonon, F. Klauser, R. Nickolaus \& R. Huisinga (Hrsg.), Kompetenz, Kognition und Neue Konzepte der beruflichen Bildung (S. 55-66). Wiesbaden: VS.

Sonntag, K.-H., \& Schaper, N. (2006). Förderung beruflicher Handlungskompetenz. In K.H. Sonntag (Hrsg.), Personalentwicklung in Organisationen (S. 230-311). Göttingen: Hogrefe.

Tett, R.P., Guterman, H. A., Bleier, A., \& Murphy, P. J. (2000). Development and content validation of a "hyperdimensional" taxonomy of managerial competence. Human Performance, 13, 205-251.

Tiemeyer, E. (2010). IT-Projekte erfolgreich managen - Handlungsbereiche und Prozesse. In E. Tiemeyer (Hrsg.), Handbuch ITProjektmanagement. Vorgehensmodelle, Managementinstrumente, Good Practices (S. 1-33). München: Hanser.
Vigenschow, U. (2008). Agile Entwicklungsteams führen. Selbstorganisation in der Praxis. HMD - Praxis der Wirtschaftsinformatik, 260, 86-94.

Wieczorrek, H., \& Mertens, P. (2011). Management von IT-Projekten. Berlin: Springer.

Zühlke, M. (2017). Diagnose-Tools gehören auf den Prüfstand. Personalwirtschaft, 8, 30-31.

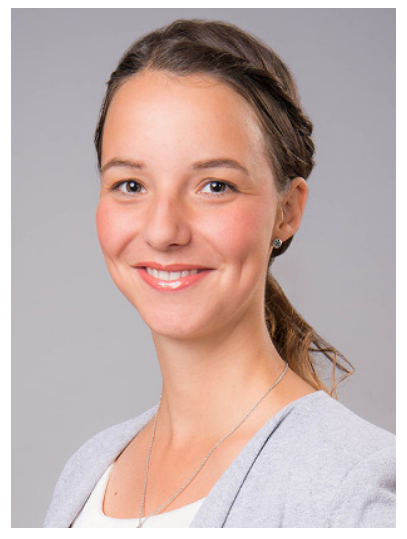

Sarah Anke studierte Betriebswirtschaft mit dem Schwerpunkt Personalmanagement an der Hochschule Merseburg. Derzeit ist sie im Bereich Personalbetreuung in einem IT-Onlineunternehmen tätig. Sie interessiert sich in Praxis und Forschung für die Rolle des (strategischen) Personalmanagements und der Mitarbeiterführung für den Erfolg eines Unternehmens. Im Rahmen ihrer Bachelorarbeit untersuchte Frau Anke in einem internationalen IT-Dienstleistungsunternehmen die Kompetenzanforderungen an Führungskräfte von IT-Entwicklerteams, deren Ergebnisse im vorliegenden Artikel zusammengefasst sind. Aufbauend auf diesen Erkenntnissen wurde der Auswahlprozess im betroffenen Unternehmen anhand eines Assessment Centers neu konzipiert, um Stellen für Führungskräfte passgenauer und langfristig erfolgreicher zu besetzen.

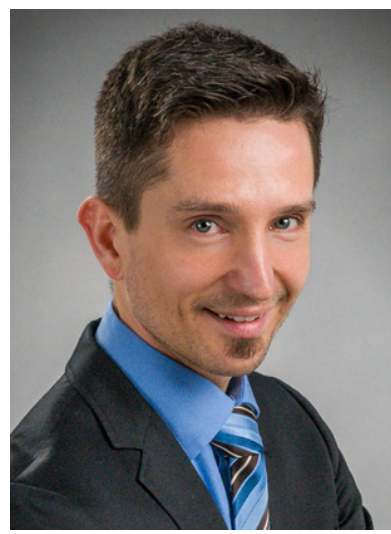

Prof. Dr. Tobias Ringeisen ist Professor für Angewandte Psychologie an der Berlin School of Economics and Law. Er beschäftigt sich in Forschung und Praxis mit der Förderung und Diagnostik von berufsrelevanten Kompetenzen im jungen Erwachsenenalter. Sein besonderes Interesse gilt dem Zusammenspiel von Persönlichkeitsmerkmalen, Lernprozessen und begleitenden Emotionen sowie Leistungsindikatoren. Weiterhin beschäftigt er sich mit der Bedeutung von Diversität in beruflichen und lernbezogenen Settings, insbesondere (inter)kultureller Diversität. Zu den genannten Themenkomplexen hat er mehr als 80 Publikationen in Form von Büchern, Zeitschriftenartikeln, Buchkapiteln und Fragebögen veröffentlicht. Zudem setzt er sich in Anwendungsprojekten mit Wirtschaft und Verwaltung für eine enge Verzahnung von Theorie und Praxis ein. Unter anderem war er als Personalentwickler, Consultant und Trainer in mehreren Unternehmen und Bundesverwaltungen sowie als wissenschaftlicher Mitarbeiter am Boston College, USA, und der Bergischen Universität Wuppertal tätig. Seit 2020 fungiert Herr Ringeisen als Präsident der Stress, Trauma, Anxiety, and Resilience Society (STAR Society; https://star-society.org/). 